\title{
ITERATIVE ALGORITHM FOR INTERFERENCE ALIGNMENT IN MULTIUSER MIMO INTERFERENCE CHANNELS
}

\author{
Heejung Yu and Youngchul Sung \\ Dept. of Electrical Engineering \\ KAIST, Daejeon, Republic of Korea \\ E-mail: hjyu@stein.kaist.ac.kr and ysung@ee.kaist.ac.kr
}

\begin{abstract}
Algorithms for designing beamforming matrices for interference alignment in multiuser time-invariant multi-input multi-output interference channels are proposed. Based on a new formulation for the necessary and sufficient condition for interference alignment, the proposed algorithm iteratively minimizes overall interference misalignment in a least squares sense. Additional modified algorithms are also considered. The convergence of the algorithm is established, and the performance is evaluated numerically. The proposed algorithms are most effective when receivers have two antennas and shows faster convergence with less complexity than the previous method with comparable sum rate performance under practical system configuration.
\end{abstract}

Index Terms-Interference channels, multi-input multi-output, interference alignment, least squares, iterative methods

\section{INTRODUCTION}

The issue of interference will become one of the most critical problems in next generation wireless communication systems since cell size is constantly being decreased and networks will be operated in interference-limited mode. In the interference-limited case, the system performance can be enhanced by proper methods of interference management. For interference channels with more than two users, a new strategy of interference alignment is an attractive solution for high signal-to-noise ratio (SNR) scenarios. The concept was originally introduced for multi-input multi-output (MIMO) $X$ channels $[1,2]$; by aligning multiple undesired signal vectors within an interference subspace at the receiver, interferencefree communication for the desired signal is possible. Interference alignment is shown to achieve the maximum degrees of freedom (DoF) in $K$-user interference channels [3]. In 3-user $M \times M$ MIMO interference channels, the maximum $\mathrm{DoF}$ is $\frac{3 M}{2}$, and a scheme that achieves for this maximum is known. For time-invariant MIMO interference channels with more than three users, however, perfect interference alignment achieving $\frac{K M}{2}$ DoF with a beamforming approach is not

This work was supported by the Korea Research Foundation Grant funded by the Korean Government (KRF-2008-220-D00079). feasible ${ }^{1}$. From an algorithmic perspective, the iterative algorithms to find the beamforming matrices that minimize interference and maximize the signal-to-interference-and-noise ratio (SINR) were proposed in [7]. The interference alignment scheme based on alternating minimization was also proposed in [8]. In [9], we introduced a least squares (LS) approach to interference alignment. In that approach, we simplified the alignment problem by setting the equivalence of two column spaces spanned by two matrices as the equivalence of the two matrices themselves. Since the equivalence of two matrices is only a sufficient condition for the equivalence of the corresponding column spaces, the feasible set for the solution in [9] is a subset of the entire feasible set, and the performance can further be enhanced by finding a solution in the entire feasible set induced by the necessary and sufficient conditions. In this paper, we generalize our previous work by considering the necessary and sufficient condition for interference alignment ${ }^{2}$. We express the condition as a single system of linear equations with dummy variables by exploiting the equivalence of two subspaces directly. This new formulation leads to a new algorithm for interference alignment that solves a least squares problem iteratively. The new algorithm shows much faster convergence than the previous method in [7] while showing the comparable performance.

\subsection{Notation}

Vectors and matrices are written in boldface with matrices in capitals. All vectors are column vectors. For a matrix $\mathbf{A}$, $\mathbf{A}^{T}, \mathbf{A}^{H}$, and $\mathbf{A}^{\dagger}$ denote the transpose, the Hermitian transpose, and the Moore-Penrose pseudo-inverse of $\mathbf{A}$, respectively. For matrices $\mathbf{A}$ and $\mathbf{B}, \mathbf{A} \otimes \mathbf{B}$ denotes the Kronecker product between the two matrices. $\operatorname{vec}(\mathbf{A})$ indicates the column vector consisting of all of the columns of $\mathbf{A} . \mathcal{C}(\mathbf{A})$ and $\mathcal{C}(\mathbf{A})^{\perp}$ represent the column space of $\mathbf{A}$ and the orthogonal

\footnotetext{
${ }^{1}$ Another approach to interference alignment using the property of irrationality of channel coefficients can achieve the maximum DoF of $\frac{K M}{2}$ [4-6]. In these references, the authors focus on the feasibility of maximum DoF and do not consider the sum rate performance. Therefore, this is out scope of this paper. [10].

${ }^{2} \mathrm{~A}$ journal version of this paper with complexity analysis was submitted
} 
complement space of $\mathcal{C}(\mathbf{A})$, respectively. $\|\mathbf{A}\|_{F}$ and $\|\mathbf{a}\|$ denote the Frobenius norm and 2-norm, respectively. $\mathbf{A}=\left[a_{l m}\right]$ means that $\mathbf{A}$ is a matrix composed of $a_{l m}$ as the $l$-th row and $m$-th column element. $\mathbf{I}_{n}$ stands for the identity matrix of size $n$. The notation $\mathbf{x} \sim \mathcal{N}(\boldsymbol{\mu}, \boldsymbol{\Sigma})$ means that $\mathbf{x}$ is complex Gaussian distributed with mean vector $\boldsymbol{\mu}$ and covariance matrix $\boldsymbol{\Sigma}$.

\section{SYSTEM MODEL}

We consider a $K$-user $M \times N$ MIMO interference channel in which $K$ transmitters with $N$ antennas transmit data streams to the corresponding receivers with $M$ antennas simultaneously. Due to the linear superposition property of wireless channels, receiver $i$ receives not only its own signal from transmitter $i$, but also signals from other undesired transmitters. Therefore, the signal vector that is received at receiver $i$ is expressed as

$$
\mathbf{y}_{i}=\mathbf{H}_{i i} \mathbf{V}_{i} \mathbf{s}_{i}+\sum_{j=1, j \neq i}^{K} \mathbf{H}_{i j} \mathbf{V}_{j} \mathbf{s}_{j}+\mathbf{n}_{i}
$$

where $\mathbf{H}_{i j}$ denotes an $M \times N$ MIMO channel matrix from transmitter $j$ to receiver $i, \mathbf{V}_{i}$ and $\mathbf{s}_{i}$ are an $N \times d_{i}$ transmit beamforming matrix and a $d_{i} \times 1$ transmit signal vector, respectively, and $\mathbf{n}_{i}$ is an $M \times 1$ complex Gaussian noise vector from distribution $\mathcal{N}\left(\mathbf{0}, \sigma^{2} \mathbf{I}\right)$. Here, we assume that the channel is time-invariant and is perfectly known to the transmitters and receivers.

The basic idea behind interference alignment is to divide the $M$ dimensional observation space at the receiver into signal and interference subspaces with $d$ and $M-d$ dimensions, respectively, and to confine all the interfering signals from the undesired transmitters within the interference subspace. If we assume that $d_{1}=d_{2}=\cdots=d_{K}=d=\frac{M}{2}$ to achieve the maximum $\mathrm{DoF}$, the interference alignment condition is given by the following.

\section{Condition 1}

$$
\begin{aligned}
\mathcal{C}\left(\mathbf{H}_{12} \mathbf{V}_{2}\right) & =\mathcal{C}\left(\mathbf{H}_{13} \mathbf{V}_{3}\right)=\cdots=\mathcal{C}\left(\mathbf{H}_{1 K} \mathbf{V}_{K}\right) \\
\mathcal{C}\left(\mathbf{H}_{21} \mathbf{V}_{1}\right)=\mathcal{C}\left(\mathbf{H}_{23} \mathbf{V}_{3}\right) & =\cdots=\mathcal{C}\left(\mathbf{H}_{2 K} \mathbf{V}_{K}\right) \\
& \vdots \\
\mathcal{C}\left(\mathbf{H}_{K 1} \mathbf{V}_{1}\right)=\mathcal{C}\left(\mathbf{H}_{K 2} \mathbf{V}_{2}\right) & =\cdots=\mathcal{C}\left(\mathbf{H}_{K K-1} \mathbf{V}_{K-1}\right)
\end{aligned}
$$

\section{ITERATIVE LEAST SQUARES ALGORITHM}

In [7], the authors proposed an interference alignment algorithm based on the idea of orthogonal complement, which was expressed as the receive beamforming matrix. This approach converts the beam design for interference alignment into a problem of solving a system of bilinear equations, which requires heavy computational cost. To circumvent this, we here exploit the structure of Condition 1 and use a linear approach to represent the condition. This new approach converts the interference alignment condition to a system of linear equations with dummy variables.

Consider the first equality in the first row in Condition 1:

$$
\mathcal{C}\left(\mathbf{H}_{12}\left[\mathbf{v}_{1}^{(2)}, \ldots, \mathbf{v}_{d}^{(2)}\right]\right)=\mathcal{C}\left(\mathbf{H}_{13}\left[\mathbf{v}_{1}^{(3)}, \ldots, \mathbf{v}_{d}^{(3)}\right]\right),
$$

where $\mathbf{v}_{k}^{(i)}$ denotes the $k$-th column of $\mathbf{V}_{i}$. The equivalence of column spaces spanned by two matrices implies that a column in one matrix is represented by a linear combination of the columns of the other. This new condition is a necessary and sufficient condition. Based on this relationship, we can rewrite (5) as

$$
\begin{aligned}
\mathbf{H}_{12} \mathbf{v}_{1}^{(2)} & =\alpha_{11}^{(13)} \mathbf{H}_{13} \mathbf{v}_{1}^{(3)}+\cdots+\alpha_{1 d}^{(13)} \mathbf{H}_{13} \mathbf{v}_{d}^{(3)}, \\
& \vdots \\
\mathbf{H}_{12} \mathbf{v}_{d}^{(2)}= & \alpha_{d 1}^{(13)} \mathbf{H}_{13} \mathbf{v}_{1}^{(3)}+\cdots+\alpha_{d d}^{(13)} \mathbf{H}_{13} \mathbf{v}_{d}^{(3)},
\end{aligned}
$$

where $\alpha_{l m}^{(13)}, l, m=1,2, \cdots, d$, are the coefficients of linear combination. Using the Kronecker product, the above multiple equalities can be rewritten as

$$
\left(\mathbf{I}_{d} \otimes \mathbf{H}_{12}\right) \operatorname{vec}\left(\mathbf{V}_{2}\right)-\left(\mathbf{A}_{13} \otimes \mathbf{H}_{13}\right) \operatorname{vec}\left(\mathbf{V}_{3}\right)=\mathbf{0},
$$

where $\mathbf{A}_{13}=\left[\alpha_{l m}^{(13)}\right]$. Collecting all equalities ${ }^{3}$ generated by Condition 1, we construct a system of linear equations with dummy variable $\mathbf{A}_{i j}$ :

$$
\tilde{\mathbf{H}} \mathbf{v}=\mathbf{0},
$$

where $\tilde{\mathbf{H}}$ is given by (10) and

$$
\mathbf{v} \triangleq\left[\operatorname{vec}\left(\mathbf{V}_{1}\right)^{T}, \operatorname{vec}\left(\mathbf{V}_{2}\right)^{T}, \cdots, \operatorname{vec}\left(\mathbf{V}_{K}\right)^{T}\right]^{T} .
$$

Therefore, a set of beamforming matrices can be designed by solving (9) with the channel information $\left\{\mathbf{H}_{i j}\right\}$ and the value of $\left\{\mathbf{A}_{i j}\right\}$.

On the other hand, Equations (6 - 7) are rewritten as

$$
\mathbf{H}_{12} \mathbf{V}_{2}=\mathbf{H}_{13} \mathbf{V}_{3} \mathbf{A}_{13}^{T} \text {. }
$$

With $V_{2}$ and $V_{3}$ given along with channel information, we can directly obtain $\mathbf{A}_{13}$ by using the left inverse and the solution is given in a closed form by

$$
\mathbf{A}_{13}=\left\{\left(\mathbf{H}_{13} \mathbf{V}_{3}\right)^{\dagger} \mathbf{H}_{12} \mathbf{V}_{2}\right\}^{T},
$$

if $\mathbf{H}_{13} \mathbf{V}_{3}$ is a tall matrix. Similarly, all other $A_{i j}$ are given by

$$
\begin{gathered}
\mathbf{A}_{1 j}=\left\{\left(\mathbf{H}_{1 j} \mathbf{V}_{j}\right)^{\dagger} \mathbf{H}_{12} \mathbf{V}_{2}\right\}^{T}, \quad j=4, \cdots, K, \\
\mathbf{A}_{i j}=\left\{\left(\mathbf{H}_{i j} \mathbf{V}_{j}\right)^{\dagger} \mathbf{H}_{i 1} \mathbf{V}_{1}\right\}^{T}, i, j=2, \cdots, K, j \neq i,
\end{gathered}
$$

when $\left\{\mathbf{V}_{j}, j=1, \cdots, K\right\}$ and $\left\{\mathbf{H}_{i j}, i, k=1, \cdots, K\right\}$ are given. Since the number of data streams is greater than that of receiver antennas, the closed form solution to $\mathbf{A}_{i j}$ always exists

${ }^{3}$ Each row in Condition 1 generates $K-2$ equalities, and we have $K$ rows in total in Condition 1. 


$$
\tilde{\mathbf{H}} \triangleq\left[\begin{array}{cccccc}
\mathbf{0} & \mathbf{I}_{d} \otimes \mathbf{H}_{12} & -\mathbf{A}_{13} \otimes \mathbf{H}_{13} & \mathbf{0} & \cdots & \mathbf{0} \\
\mathbf{0} & \mathbf{I}_{d} \otimes \mathbf{H}_{12} & \mathbf{0} & -\mathbf{A}_{14} \otimes \mathbf{H}_{14} & \cdots & \mathbf{0} \\
\vdots & \vdots & \vdots & \vdots & \vdots & \vdots \\
\mathbf{0} & \mathbf{I}_{d} \otimes \mathbf{H}_{12} & \mathbf{0} & \cdots & \cdots & -\mathbf{A}_{1 K} \otimes \mathbf{H}_{1 K} \\
\mathbf{I}_{d} \otimes \mathbf{H}_{21} & \mathbf{0} & -\mathbf{A}_{23} \otimes \mathbf{H}_{23} & \mathbf{0} & \cdots & \mathbf{0} \\
\mathbf{I}_{d} \otimes \mathbf{H}_{21} & \mathbf{0} & \mathbf{0} & -\mathbf{A}_{24} \otimes \mathbf{H}_{24} & \cdots & \vdots \\
\vdots & \vdots & \vdots & \vdots & \vdots & -\mathbf{A}_{2 K} \otimes \mathbf{H}_{2 K} \\
\mathbf{I}_{d} \otimes \mathbf{H}_{21} & \mathbf{0} & \mathbf{0} & \cdots & \cdots & \vdots \\
\vdots & \vdots & \vdots & \vdots & \vdots & \mathbf{0} \\
\mathbf{I}_{d} \otimes \mathbf{H}_{K 1} & -\mathbf{A}_{K 2} \otimes \mathbf{H}_{K 2} & \mathbf{0} & \cdots & \cdots & \vdots \\
\vdots & \vdots & \vdots & \vdots & \vdots & \mathbf{0}
\end{array}\right],
$$

\subsection{Basic Algorithm}

Based on the above results, we propose a new algorithm for beam design for interference alignment. The overall structure of the proposed algorithm is as follows. First, we find a solution $\left\{\mathbf{V}_{j}\right\}$ to (9) with a given initial value for $\left\{\mathbf{A}_{i j}\right\}$. With the obtained beamforming matrices, we update the linear coefficient matrices $\left\{\mathbf{A}_{i j}\right\}$ using (13 - 15). By iterating this process until it converges, we obtain a solution to interference alignment. The second step of finding $\left\{\mathbf{A}_{i j}\right\}$ is simple, and its solution is given in closed form when $\left\{\mathbf{H}_{i j} \mathbf{V}_{j}\right\}$ are tall matrices. In the first step, however, the linear system (9) can be overdetermined depending on $K, M, N$, and $d$. When $d_{1}=\cdots=d_{K}=M / 2$ and $N=M$, for example, the size of $\tilde{\mathbf{H}}$ is $K(K-2) \frac{M^{2}}{2} \times K \frac{M^{2}}{2}$ and the linear system is overdetermined for $K \geq 4$ with additional constraint on $\mathbf{v}$. Since an exact solution does not exist in such cases, we apply a least squares approach to (9). Then, the problem can be formulated as

$$
\hat{\mathbf{v}}=\underset{\|\mathbf{v}\|=1}{\arg \min }\|\tilde{\mathbf{H}} \mathbf{v}\|
$$

where $\|\tilde{\mathbf{H}} \mathbf{v}\|$ can be considered as the norm of the overall interference misalignment. The solution to (16) is known to be the eigenvector associated with the smallest eigenvalue. Furthermore, the eigenvector corresponding to extreme eigenvalues can easily be obtained by the power method [11]. Thus, the proposed algorithm is described as follows.

\section{Algorithm 1 (Basic iterative least squares algorithm)}

1. Initialize $\mathbf{A}_{i j}=\mathbf{I}$.

2. Construct $\tilde{\mathbf{H}}$ with $\left\{\mathbf{H}_{i j}\right\}$ and $\left\{\mathbf{A}_{i j}\right\}$.

3. Obtain $\hat{v}$ by solving (16) for (9) in the least squares sense using the power method.

3.1. Initialization: $\mathbf{b}=[1,0, \cdots, 0], \mathbf{R}=c \mathbf{I}-\tilde{\mathbf{H}}^{H} \tilde{\mathbf{H}}$, where constant $c$ is chosen so that $c \mathbf{I}-\tilde{\mathbf{H}}^{H} \tilde{\mathbf{H}}$ is a positive definite matrix.

3.2. $\mathrm{z}=\mathbf{R b}$.

3.3. $\mathbf{b}=\frac{\mathbf{z}}{\|\mathbf{z}\|}$
3.4. Iterate 3.1 to 3.3 until it converges.

4. Obtain $\left\{\mathbf{V}_{j}\right\}$ by reshaping $\hat{\mathbf{v}}$ from Step 3 .

5. Determine $\left\{\mathbf{A}_{i j}\right\}$ using $\left\{\mathbf{V}_{j}\right\}$ from Step 4 based on (13 15).

6. Iterate Steps 2 to 5 until it converges.

\subsection{Modified Algorithms}

We can additionally consider the modification of the proposed algorithm as in [9]. Though Algorithm 1 finds the solution effectively, it has the global transmit power constraint $\|\mathbf{v}\|=$ 1 instead of the individual power constraint $\left\|\mathbf{v}_{k}^{(i)}\right\|=1$ for all $k$ and $i$. Hence, unfair power allocation to each transmitter and each data stream may occur depending on the realization of channel matrices. To avoid this problem, we consider the following modification.

\section{Algorithm 2 (With individual power constraint)}

$4^{\prime}$. Obtain $\left\{\mathbf{V}_{j}\right\}$ by reshaping and normalizing $\hat{\mathbf{v}}$ from Step 3.

$4^{\prime}$.1. Obtain $\left\{\mathbf{V}_{j}=\left[\mathbf{v}_{1}^{(j)}, \cdots, \mathbf{v}_{d}^{(j)}\right]\right\}$ with $\hat{\mathbf{v}}$.

$4^{\prime}$.2. Normalize $\mathbf{v}_{k}^{(j)}$ such that $\left\|\mathbf{v}_{k}^{(j)}\right\|=1, k=1, \cdots d$, $j=1, \cdots, K$.

All other steps in Algorithm 2 except Step 4 are the same as in Algorithm 1. Note that the column-wise scaling of beamforming matrices does not change the linear subspace itself. Thus, with this additional scaling step to Algorithm 1, we accomplish both the minimization of the overall interference misalignment and the individual norm constraint.

So far, we have considered the minimization of interference misalignment only. Since the final goal of interference alignment is to increase the sum rate, the desired signal power should be incorporated in the algorithm. Such an algorithm (called Algorithm 3 here) can be constructed by incorporating the signal power $\left\|\mathbf{H}_{i i} \mathbf{V}_{i}\right\|_{F}$. The modified optimization problem is given by

$$
\min \mathbf{v}^{H}\left(\tilde{\mathbf{H}}^{H} \tilde{\mathbf{H}}-\gamma \boldsymbol{\Phi}^{H} \boldsymbol{\Phi}\right)_{+} \mathbf{v}
$$


subject to $\left\|\mathbf{D}_{11} \mathbf{v}\right\|=\cdots=\left\|\mathbf{D}_{1 d} \mathbf{v}\right\|=\cdots=\left\|\mathbf{D}_{K d} \mathbf{v}\right\|=$ $P$ with a weighting factor $\gamma>0$ for the signal power. Here, $\boldsymbol{\Phi}$ is defined as $\boldsymbol{\Phi} \triangleq \operatorname{diag}\left(\mathbf{I}_{d} \otimes \mathbf{H}_{11}, \cdots, \mathbf{I}_{d} \otimes \mathbf{H}_{K K}\right)$ so that $\mathbf{v}^{H} \boldsymbol{\Phi}^{H} \mathbf{\Phi} \mathbf{v}=\|\mathbf{\Phi} \mathbf{v}\|^{2}=\sum_{k=1}^{K}\left\|\mathbf{H}_{k k} \mathbf{V}_{k}\right\|_{F}^{2}$. Here, we can also optimize the weighting factor $\gamma$ depending on the compromise between signal power maximization and interference misalignment minimization. Throughout this paper, we set $\gamma=\frac{1}{S N R}$. The implementation of Algorithm 3 is similar to Algorithm 2.

For maximization of the sum rate, the angle between the signal and interference subspaces is one of the most important factors. If a zero-forcing method is used for receiver processing, the desired signal, $\mathbf{H}_{i i} \mathbf{V}_{i}$, is projected onto the orthogonal complement subspace of the interference subspace, $\mathcal{C}\left(\mathbf{H}_{i j} \mathbf{V}_{j}\right)^{\perp}$. Therefore, we can obtain a higher signal-tointerference ratio (SIR) when the signal and interference subspaces are nearly orthogonal to each other. In that sense, one of our objectives can be to minimize an inner product of signal and interference components, $\sum_{i} \sum_{j \neq i}\left\|\mathbf{V}_{i}^{H} \mathbf{H}_{i i}^{H} \mathbf{H}_{i j} \mathbf{V}_{j}\right\|_{F}$. Given $\{\mathbf{A} i j\}$, the problem to find $\hat{\mathbf{v}}$ is given by

$$
\begin{array}{cc}
\min & \mathbf{v}^{H}\left(\tilde{\mathbf{H}}^{H} \tilde{\mathbf{H}}-\gamma \Phi^{H} \Phi\right)_{+} \mathbf{v}+\gamma^{\prime} t \\
\text { s.t. } & \|\mathbf{v}\|^{2}=1 \\
& \sum_{i} \sum_{j \neq i} \sum_{k=1}^{d}\left|\mathbf{v}^{H} \mathbf{S}_{i, k} \mathbf{H}_{i i}^{H} \mathbf{H}_{i j} \mathbf{S}_{j, k} \mathbf{v}\right| \leq t
\end{array}
$$

where $\mathbf{S}_{i, k}$ denotes a matrix extracting $\mathbf{v}_{k}^{(i)}$ from $\mathbf{v}$. In this paper, we set $\gamma^{\prime}=\frac{1}{S N R}$. This problem is in the complicated form so the solution should be found with a numerical optimization method ${ }^{4}$. By replacing Step 3 of the previous algorithm with the procedure of solving $(18-20)$, we can devise Algorithm 4.

\section{CONVERGENCE}

In this section, the convergence of the proposed iterative algorithm for interference alignment is established.

Theorem 1 (Convergence) The iterative least squares algorithms which are based on Equations (9) and (13-15) converge.

Proof: Define $\tilde{\mathbf{H}}\left(\left\{\mathbf{A}_{i j}[n-1]\right\}\right)$ as the matrix $\tilde{\mathbf{H}}$ based on $\left\{\mathbf{A}_{i j}[n-1]\right\}$. Then, $\hat{\mathbf{v}}[n]$ at the $n$-th iteration is given by

$$
\hat{\mathbf{v}}[n]=\underset{\mathbf{v}}{\arg \min }\left\|\tilde{\mathbf{H}}\left(\left\{\mathbf{A}_{i j}[n-1]\right\}\right) \mathbf{v}\right\|
$$

under the constraint on $\mathbf{v}$ of each algorithm. Then,

$$
\begin{aligned}
I[n] & \triangleq\left\|\tilde{\mathbf{H}}\left(\left\{\mathbf{A}_{i j}[n-1]\right\}\right) \hat{\mathbf{v}}[n]\right\|, \\
& \geq\left\|\tilde{\mathbf{H}}\left(\left\{\mathbf{A}_{i j}[n]\right\}\right) \hat{\mathbf{v}}[n]\right\| .
\end{aligned}
$$

\footnotetext{
${ }^{4}$ In this paper, we used "fmincon" in the MATLAB optimization toolbox to evaluate the performance
}

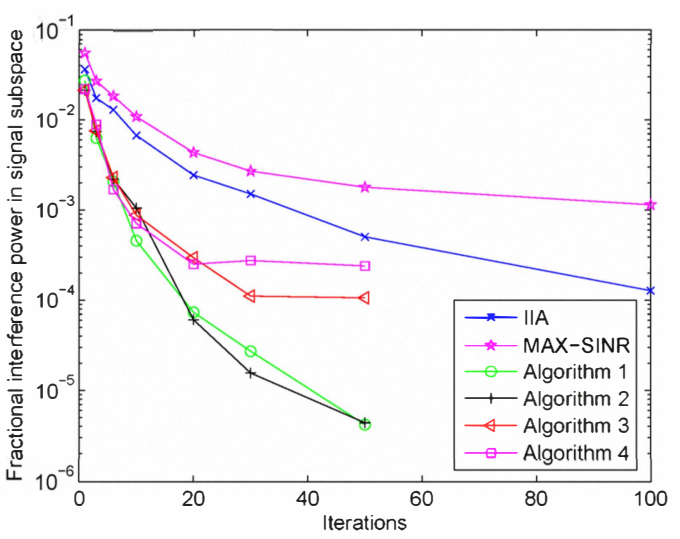

Fig. 1. Total interference leakage into the signal space with respect to iteration number at $20 \mathrm{~dB}$ SNR when $M=2, N=$ $2, K=3$, and $d=1$.

The inequality (23) holds because $\left\{\mathbf{A}_{i j}[n]\right\}$ itself is the least squares solution that minimizes

$$
\begin{aligned}
\Delta_{i j}[n] & \triangleq\left\|\mathbf{H}_{i j^{\prime}} \hat{\mathbf{V}}_{j^{\prime}}[n]-\mathbf{H}_{i j} \hat{\mathbf{V}}_{j}[n] \mathbf{A}_{i j}\right\|_{F} \\
& =\left\|\left(\mathbf{I} \otimes \mathbf{H}_{i j^{\prime}}\right) \operatorname{vec}\left(\hat{\mathbf{V}}_{j^{\prime}}[n]\right)-\left(\mathbf{A}_{i j} \otimes \mathbf{H}_{i j}\right) \operatorname{vec}\left(\hat{\mathbf{V}}_{j}[n]\right)\right\|
\end{aligned}
$$

for given $\hat{\mathbf{v}}[n]=\operatorname{vec}\left(\left[\hat{\mathbf{V}}_{1}[n], \cdots, \hat{\mathbf{V}}_{K}[n]\right]\right)$, and $\|\tilde{\mathbf{H}} \hat{\mathbf{v}}[n]\|^{2}=$ $\sum_{i, j} \Delta_{i j}^{2}[n]$ (See (10)). From the fact that

$$
\hat{\mathbf{v}}[n+1]=\underset{\mathbf{v}}{\arg \min }\left\|\tilde{\mathbf{H}}\left(\left\{\mathbf{A}_{i j}[n]\right\}\right) \mathbf{v}\right\|,
$$

we have

$I[n+1]=\left\|\tilde{\mathbf{H}}\left(\left\{\mathbf{A}_{i j}[n]\right\}\right) \hat{\mathbf{v}}[n+1]\right\| \leq\left\|\tilde{\mathbf{H}}\left(\left\{\mathbf{A}_{i j}[n]\right\}\right) \hat{\mathbf{v}}[n]\right\|$.

Combining (23) and (25), we obtain $I[n+1] \leq I[n]$. Thus, the norm of misaligned interference decreases monotonically with the number iteration. Since the norm of interference misalignment is lower bounded by zero, the algorithms eventually converge by the monotone convergence theorem.

Theorem 1 shows the convergence of the proposed iterative algorithms via the convergence of the norm of interference misalignment. Next, we compare the convergence speed of the proposed algorithms and the previous methods in [7]. The total interference leakage into the signal subspace defined in [7] instead of the interference misalignment is used for performance measure. It is seen in Fig. 1 that the proposed algorithms converges much faster than the previous methods. In Fig. 1, "IIA" minimizing interference and "MAX-SINR" maximizing SINR donote the iterative interference algorithms in [7]. Note that the leakage levels of Algorithms 3 and 4 are higher than those of Algorithms 1 and 2 because of the additional design goal of increasing the signal power and minimizing the inner product. However, it will be shown later that this is not detrimental to the sum rate performance. 


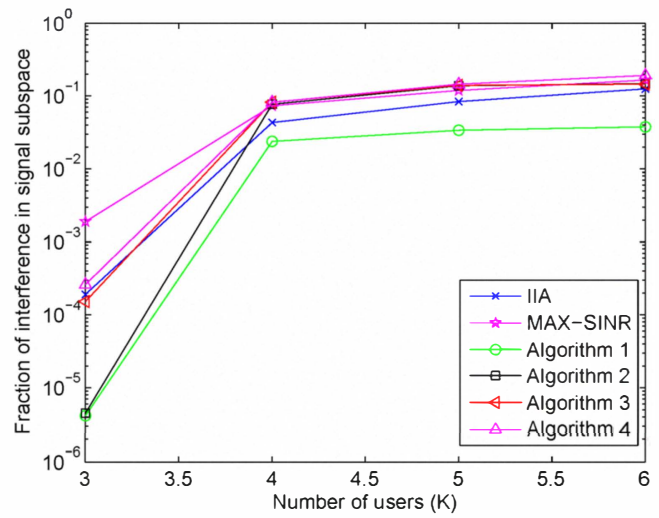

Fig. 2. Total fractional leakage into the signal space at $20 \mathrm{~dB}$ SNR when $M=2, N=2$, and $d=1$.
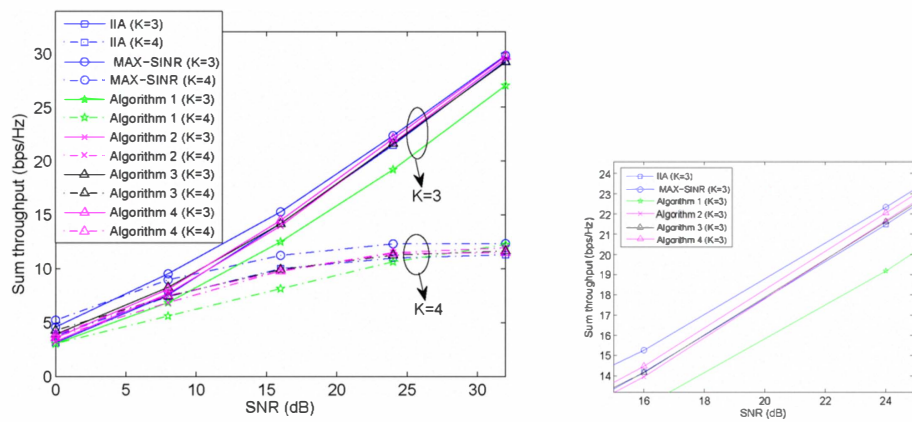

Fig. 3. Sum rate when $M=2, N=2$, and $d=1$.

\section{NUMERICAL RESULTS}

The performance of the proposed algorithms was evaluated numerically in terms of the interference leakage into the signal space and sum rate. Fig. 2 shows the interference leakage into the signal subspace for all six algorithms including the previous algorithms in [7] after the convergence for the cases of $M=2, N=2$, and $d=1$ with $20 \mathrm{~dB}$ SNR. As expected, Algorithm 1 yields the least leakage into the signal subspace because of having the largest amount of freedom in choosing v. MAX-SINR and Algorithms 3 and 4 show higher interference leakage since these have additional objectives to maximize the signal power and minimize the inner product.

Finally, we evaluated the sum rate performance of the algorithms. Fig. 3 shows the sum rate performance for the case of $M=2, N=2$, and $d=1$. In this case, all six algorithms show a linear increase of sum rate with respect to (w.r.t.) SNR for $K=3$. From this observation, we can see that all algorithms guarantee the maximum degrees of freedom. When $K=4$, the sum rates are saturated due to infeasibility of interference alignment. Among the proposed algorithms, Algorithm 4 shows the highest sum rate since it considers three factors, interference misalignment, signal power, and inner product, which are related to the sum rate performance. Though MAX-SINR shows the highest sum rate, it requires lots of iterations and computational complexity. The proposed methods have faster convergence and lower complexity than the previous ones while maintaining negligible performance loss.

\section{CONCLUSION}

We have considered the interference alignment for $K$-user time-invariant MIMO interference channels. We have proposed new interference alignment algorithms based on an iterative LS approach. The proposed algorithm is most effective for the most practical case in which the receivers have two antennas. We have established the convergence of the proposed algorithms. It has been shown that the proposed algorithms have fast convergence speed while maintaining comparable sum rate performance.

\section{REFERENCES}

[1] M. Maddah-Ali, A. Motahari, and A. Khandani, "Signaling over MIMO multi-base systems - combination of multiple access and broadcast schemes," in Proc. ISIT, pp. $2104-2108$, Seattle, WA, July 2006.

[2] S. Jafar and S. Shamai, "Degrees of freedom for the MIMO $X$ channel," IEEE Trans. Inform. Theory, vol. 54, pp. 151 - 170, Jan. 2008.

[3] V. R. Cadambe and S. A. Jafar, "Interference alignment and degrees of freedom of the $K$-user interference channel," IEEE Trans. Inform. Theory, vol. 54, pp. 3425 - 3441, Aug. 2008.

[4] A. S. Motahari, S. O. Gharan, M. A. Maddah-Ali, and A. K. Khandani, "Forming pseudo-MIMO by embedding infinite rational dimensions along a single real line: removing barriers in achieving the DoFs of single antenna systems," ArXiv pre-print cs.IT/0908.2282, Aug. 2009.

[5] R. Erkin and E. Ordentlich, "On the degrees-of-freedom for the $K$-user Gaussian interference channel," ArXiv pre-print cs.IT/0901.1695, Jan. 2009.

[6] A. Ghasemi, A. S. Motahari, and A. K. Khandani, "Interference alignment for the $K$ user MIMO interference channel," ArXiv pre-print cs.IT/0909.4604, Sept. 2009.

[7] K. Gomadam, V. R. Cadambe, and S. A. Jafar, "Approaching the capacity of wireless networks through distributed interfernce alignment," ArXiv pre-print cs.IT/0803.3816, Mar. 2008.

[8] S. W. Peters and R. W. Heath, "Interference alginment via alternating minimization," in Proc. IEEE ICASSP, Taipei, Taiwan, Apr. 2009.

[9] H. Yu, J. Park, Y. Sung, and Y. H. Lee, "A least squares approach to joint beam design for interference alignment in multiuser interference channels," in Proc. IEEE SPAWC, Perugia, Italy, June 2009.

[10] H. Yu and Y. Sung, "Least squares approach to joint beam design for interference alignment in multiuser multi-input multioutput interference channels," submitted to IEEE Trans. Signal Process., Dec. 2009.

[11] G. H. Golub and C. F. V. Loan, Matrix Computations. Baltimore, MD: 2nd Edition, Johns Hopkins University Press, 1996. 